\title{
Crystallization study of Te-Bi-Se glasses
}

\author{
MANISH SAXENA* and P K BHATNAGAR ${ }^{\dagger}$ \\ Department of Sciences and Humanities, Moradabad Institute of Technology, Moradabad 244 001, India \\ ${ }^{\dagger}$ Department of Electronic Science, University of Delhi, New Delhi 110 021, India
}

MS received 16 December 2002; revised 29 April 2003

\begin{abstract}
Crystallization studies are carried out under non-isothermal conditions with samples heated at several uniform rates. The dependence of the glass transition temperature $\left(T_{\mathrm{g}}\right)$, the crystalline temperature $\left(T_{c}\right)$ and the peak temperature of crystallization $\left(T_{p}\right)$ on the composition and heating rate $(\beta)$ has been studied. For a memory/switching material, the thermal stability and ease of glass formation are of crucial importance. The glass transition temperature, $T_{\mathrm{g}}$, increases slightly with the variation of $\mathrm{Bi}$ content. From the heating rate dependence of $T_{\mathrm{g}}$, the activation energy for glass transition $\left(E_{\mathrm{t}}\right)$ has been evaluated. The results are discussed on the basis of Kissinger's approach and are interpreted using the chemically ordered network model (CONM).
\end{abstract}

Keywords. Thermal stability; chalcogenide glasses; glass forming ability; glass transition temperature.

\section{Introduction}

There has been considerable interest in amorphous semiconductors because of their interesting physical properties as well as their wide technological applications including threshold and memory switching. Threshold switches are made in those glasses near the centre of the glass-forming region where the glasses are stable and show little or no tendency to crystallize when heated or cooled slowly. Memory switches come from the boundaries of the glassforming region where the glasses are more prone to crystallization. In chalcogenide glass systems, the glasses which exhibit no exothermic crystallization reaction above the glass transition temperature, $T_{\mathrm{g}}$, could possibly be used in threshold switching systems whereas glasses exhibiting an endothermic crystallization reaction above $T_{\mathrm{g}}$ show a memory type of switching (Onozuka and Oda 1988). It is thought for long that memory or threshold behaviour of chalcogenide glasses is determined essentially by their crystallization ability. Those which are prone to easy devitrification, show memory and stable glasses exhibit threshold behaviour. However, the recent results on the switching behaviour of several chalcogenide glasses have shown that in addition to crystallization tendency, there are other important aspects such as ON-state current, thermal diffusivity, network rigidity, etc which decide whether the sample will show memory or threshold behaviour. It has been shown that the amorphous films with composition outside the glass-forming region are more suitable for memory devices because of their fast crystallization during the application of a suitable electric

\footnotetext{
*Author for correspondence
}

pulse or by the heat of a focused laser beam. The kinematical studies are always connected with the concept of the activation energy. The values of these studies in glass crystallization phenomena are associated with the nucleation and growth processes, which dominate the devitrification of most glassy solids. In general, separate activation energies must be identified with individual nucleation and growth steps in a transformation, although they usually have been combined into activation energy representative of the overall crystallization process. Among amorphous chalcogenide alloys, selenium based melt are characterized by high viscosity (Khan et al 1997; Ilyas et al 2000). This feature favours the glass formation in bulk form by air-quenching or water-quenching as well as in evaporated thin film forms. Since tellurium based melts with the same elements generally have low viscosity, a high cooling rate is required to prevent nucleation and growth during quenching and to obtain bulk glasses. The tellurium alloys have often been used for the active layer of those devices because they have low melting point. Te-based alloys which contain a small amount of $\mathrm{Ge}, \mathrm{Bi}, \mathrm{Sb}$ or As exhibit fast crystallization processes after switching. However, these alloys present several problems like segregation and low crystallization temperature. The segregation limits the reversible transition between crystalline and amorphous states, because in every crystallization process, the compositional deviation is likely to continue in the active layer. A ternary solid alloy with relatively low melting point may be the answer to the above problems, because no compositional changes occur in a solid solution when it is cycled between amorphous and crystalline states. In this paper, we report our studies on $\mathrm{Te}-\mathrm{Bi}-\mathrm{Se}$ ternary glasses. A fundamental observation recently made for the rapidly quenched metal alloys is that they do not usually indicate 
a sharp glass liquid transition characteristic of amorphous material (Gosain et al 1991). The studies of crystalline kinetics of a glass upon heating can be performed in several different ways. In calorimetric measurements, two basic methods can be used: isothermal and non-isothermal. In the isothermal method, the sample is brought quickly to a temperature above the glass transition temperature, $T_{\mathrm{g}}$, and the heat evolved during the crystallization process at a constant temperature is recorded as a function of time. In the nonisothermal method, the sample is heated at a fixed rate $(\beta)$ and the heat evolved is recorded as a function of temperature or time (Saxena et al 2003). The main factor, which leads to the stability of amorphous phase of a compound near room temperature, is its glass transition temperature, $T_{\mathrm{g}}$, if the melting temperature of the compound is high, and the glass transition temperature, $T_{\mathrm{g}}$, of it is expected to be considerably higher than room temperature. For phase change optical switching systems, it is important to optimize various parameters like the glass transition temperature, $T_{\mathrm{g}}$, the crystallization temperature, $T_{\mathrm{c}}$ and the melting temperature, $T_{\mathrm{m}}$, by varying the composition and heating rate (Saxena et al 2001). Crystallization studies are carried out under non-isothermal conditions with samples heated at several uniform rates.

The activation energy plays a dominant role in deciding the utility of the material for the specific purpose, here, storage. The value of this energy in glass crystalline phenomena is associated with nucleation and growth processes that dominate the devitrification of most glassy solids (Tokunaga et al 1989). One of the most important aspects of the study of glasses is the composition dependence of properties. In order to understand the composition dependence, various models, viz. the random covalent network model (RCNM), the chemically ordered network model (CONM) and recently the constraints model and the structural phase transition model are generally used. The dependence of the glass transition temperature $\left(T_{\mathrm{g}}\right)$, the crystalline temperature $\left(T_{\mathrm{c}}\right)$ and the peak temperature of crystallization $\left(T_{\mathrm{p}}\right)$ on the composition and heating rate has been studied in the present work. From the heating rate dependence of $T_{\mathrm{g}}$, the activation energy for glass transition $\left(E_{\mathrm{t}}\right)$ has been evaluated.

\section{Experimental}

For preparation of Te-Bi-Se glasses, high purity $(5 \mathrm{~N})$ elements in appropriate atomic percentages were weighted into the quartz ampoules ( $8 \mathrm{~mm}$ diameter). The contents of the ampoules were sealed off in a vacuum of $10^{-5}$ torr and heated in a temperature-controlled furnace at around $900^{\circ} \mathrm{C}$ for $24 \mathrm{~h}$. The ampoules were rotated frequently to ensure homogenization. The ampoules were then rapidly quenched in ice-water mixture to obtain the glasses. The quenched samples were removed from the ampoules by dissolving the ampoules cautiously in a mixture of $\mathrm{HF}+$ $\mathrm{H}_{2} \mathrm{O}_{2}$ for about $20 \mathrm{~h}$. The amorphous nature of the result- ing glassy alloys was verified by X-ray diffraction. A RIGAKU DTA calorimeter was used to record the thermograms to determine the thermal behaviour of samples. For scanning the samples for the variation of glass transition temperature, $T_{\mathrm{g}}$, with heating rates, the samples were scanned at different heating rates ranging from $10 \mathrm{~K} / \mathrm{min}$ to $20 \mathrm{~K} / \mathrm{min}$ in the temperature range $20-800^{\circ} \mathrm{C}$.

\section{Results and discussion}

The transformation to a glass does not take place at one, strictly defined temperature, but within a temperature range, representing the transformation region (Vacher et al 1974). The width of the later depends on the properties of the material studied (low-temperature edge) and on the thermal history of the sample (high-temperature edge). The single endothermic glass transition peak indicates the homogeneity of the glass. $T_{\mathrm{g}}$ represents the 'strength' or the 'rigidity' of the glass structure. Therefore, drastic changes in $T_{\mathrm{g}}$ cannot be expected by increase in $\mathrm{Bi}$ content, which results in isostructural units of nearly same bond strength. The slight increase in $T_{\mathrm{g}}$ observed is probably due to the increase in mean molecular weight of the glasses with increasing $\mathrm{Bi}$ content. The observed increase is attributed either to the increase in the effective molecular weight with increasing $\mathrm{Bi}$ content or to the increase in concentration of long polymer chains.

In the present $\mathrm{Te}-\mathrm{Bi}-\mathrm{Se}$ glass system, the glass transition temperature was found to increase with the increase in $\mathrm{Bi}$ content as well as with the increase in heating rates from $10 \mathrm{~K} / \mathrm{min}$ to $20 \mathrm{~K} / \mathrm{min}$. This may be attributed to the fact that when heating rate is high, the system does not get sufficient time for nucleation and crystallization. By the time crystallization starts taking place, the temperature goes up owing to the higher heating rates. The variations of $T_{\mathrm{g}}$ as a function of $\mathrm{Bi}$ content, for different heating rates, in the $\mathrm{Te}-\mathrm{Bi}-\mathrm{Se}$ system have been shown in figure 1.

These observations can be explained to some extent with the help of CONM wherein the formation of heteropolar bonds is favoured over the formation of homopolar bonds. In the Te-Bi-Se system the various bonds involved are $\mathrm{Bi}-\mathrm{Te}, \mathrm{Bi}-\mathrm{Se}, \mathrm{Te}-\mathrm{Se}, \mathrm{Se}-\mathrm{Se}, \mathrm{Te}-\mathrm{Te}$ etc. When the atomic percentage of $\mathrm{Bi}$ is increased in $\mathrm{Te}-\mathrm{Bi}-\mathrm{Se}$ glass system, $\mathrm{Bi}$ is expected to combine preferably with $\mathrm{Se}$ because the bond energy of $\mathrm{Bi}-\mathrm{Se}(170.4 \mathrm{~kJ} / \mathrm{mol})$ is greater than that of $\mathrm{Bi}-\mathrm{Te}(125.6 \mathrm{~kJ} / \mathrm{mol})$. This results in decreasing Se-Se bonds. This explains the increase in $T_{\mathrm{g}}$ with the increase in $\mathrm{Bi}$ content due to the formation of large number of heteropolar $\mathrm{Bi}-\mathrm{Se}$ bonds and decrease in homopolar Se-Se, Te-Te and Te-Se bonds. The results are similar to those obtained by earlier workers (Rahman et al 1991).

Two approaches are used to discuss the dependence of $T_{\mathrm{g}}$ on the heating rate $\beta$ : 
One is the empirical relationship of the form (Lasocka 1976)

$$
T_{\mathrm{g}}=A+B \log \beta,
$$

where $A$ and $B$ are constants for a given glass composition and $\beta$ the heating rate, holds good for all the samples of Te-Bi-Se glass system. The values of $B$ are found to be different for different glass compositions, indicating that $\mathrm{Te}-\mathrm{Bi}-\mathrm{Se}$ alloy undergoes structural changes for different $\mathrm{Bi}$ concentrations, because the values of $B$ is an indication of the response of the configurational changes within the glass transition region.

The other approach that is commonly used in analysing crystallization data in DTA/DSC analysis for the evaluation of the activation energy for glass transition, $E_{\mathrm{t}}$, was developed by Kissinger (1956), which is expressed as

$$
\ln \left(T_{\mathrm{g}}^{2} / \beta\right)+\text { const }=E_{\mathrm{t}} / k T_{\mathrm{g}},
$$

where $k$ is Boltzmann's constant.

The plots of $T_{\mathrm{g}}$ vs $\beta$ (figure 1$)$ and $\ln \left(T_{\mathrm{g}}^{2} / \beta\right)$ vs $10^{3} / T_{\mathrm{g}}$ (figure 2) are seen to be linear for these glasses up to a heating rate of $20 \mathrm{~K} / \mathrm{min}$. The values of activation energy, $E_{\mathrm{t}}$, obtained using the plots of figure 2 are listed in table 1. Activation energy, $E_{\mathrm{t}}$, for the glass transition is found to decrease from $1.896 \mathrm{eV}$ to $1.565 \mathrm{eV}$ with the increase in $\mathrm{Bi}$ content for these samples from $28 \%$ to $38 \%$. The variation of activation energy for glass transition, $E_{\mathrm{t}}$, with $\mathrm{Bi}$ content is given in figure 4 .

The Kissinger equation can be approximated by the form (Afify 1990)

$$
\ln \beta=-E_{\mathrm{t}} / k T_{\mathrm{g}}+\text { constant. }
$$

Figure 3 shows the plots of $\ln \beta$ vs $10^{3} / T_{\mathrm{g}}$ for different compositions of $\mathrm{Te}-\mathrm{Bi}-\mathrm{Se}$ system, which are seen to be linear for these glasses. The activation energy for glass transition, $E_{\mathrm{t}}$, deduced using this relation is found to

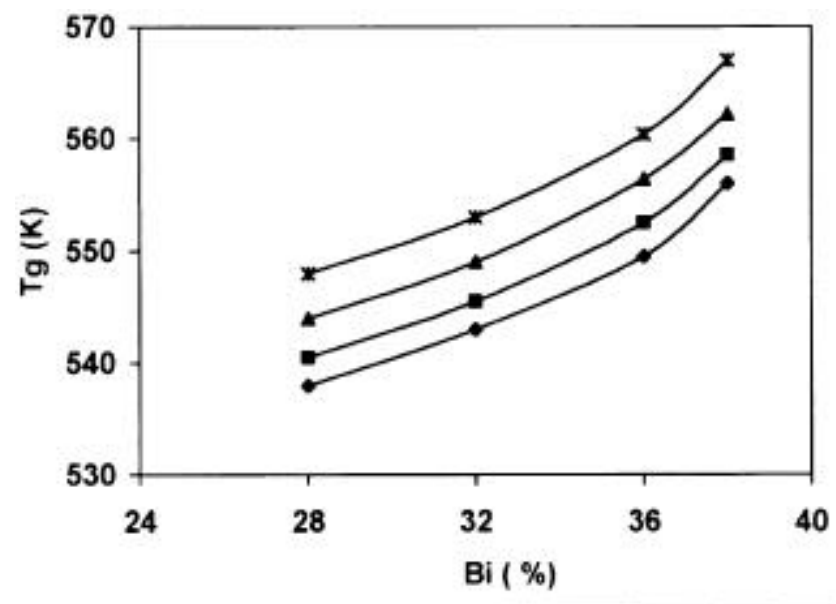

Figure 1. Variation of $T_{\mathrm{g}}$ as a function of $\mathrm{Bi}$ at various heating rates. decrease from $1.982 \mathrm{eV}$ to $1.652 \mathrm{eV}$ with the increase in $\mathrm{Bi}$ content from $28 \%$ to $38 \%$. The variation of activation energies, deduced by using (3) with $\mathrm{Bi}$ content is shown in figure 4.

From the comparison of these two results, it is evident that the two deduced values, so obtained by using (2) and (3), are in good agreement with each other. This means that one can use either of the two relations to calculate glass transition activation energy, $E_{\mathrm{t}}$.

Table 1. Values of activation energies using two relations.

\begin{tabular}{lcc}
\hline $\mathrm{Bi}(\%)$ & $E_{\mathrm{t}}(\mathrm{eV})(2)$ & $E_{\mathrm{t}}(\mathrm{eV})(3)$ \\
\hline 28 & 1.895 & 1.982 \\
32 & 1.665 & 1.749 \\
36 & 1.610 & 1.698 \\
38 & 1.565 & 1.657 \\
\hline
\end{tabular}

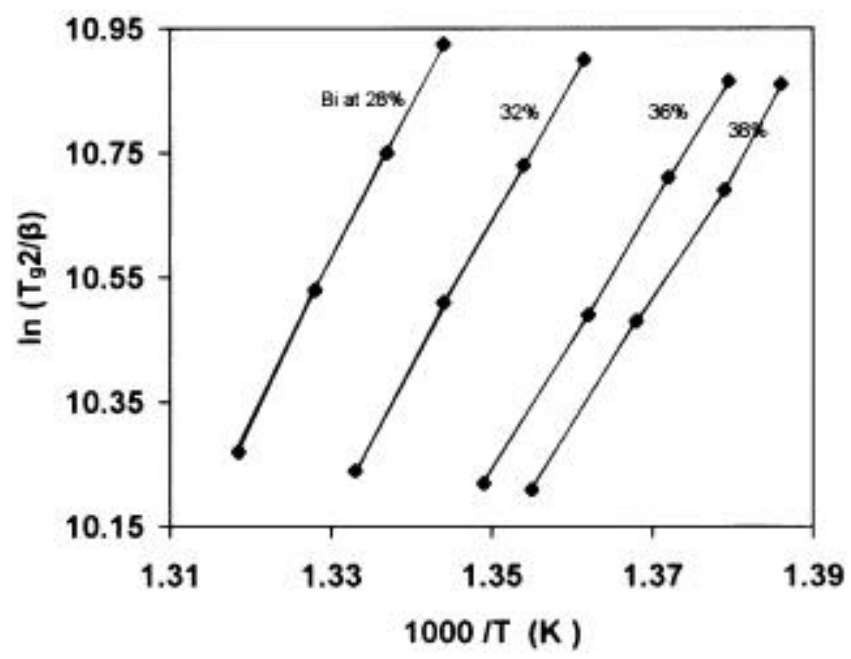

Figure 2. Relation between $\ln \left(T_{\mathrm{p}}^{2} / \beta\right)$ and $10^{3} / T_{\mathrm{p}}$ for various compositions.

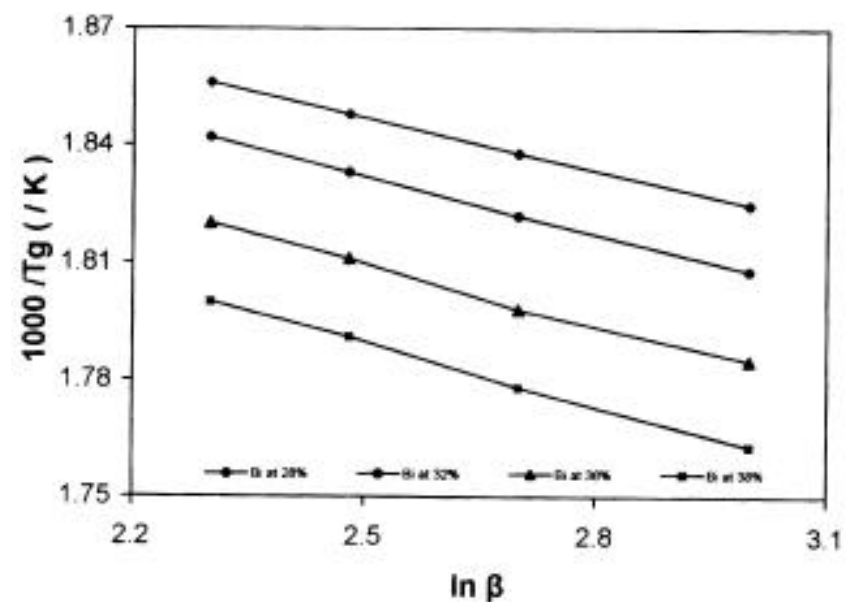

Figure 3. $10^{3} / T_{\mathrm{g}}$ vs $\ln \beta$ for different Bi contents. 


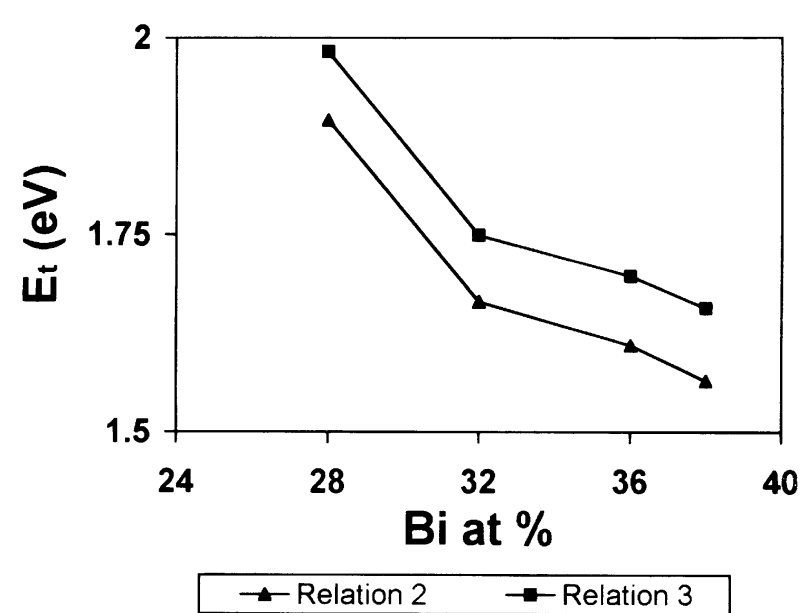

Figure 4. Variation of activation energy, $E_{\mathrm{t}}$, with Bi content.

\subsection{Glass forming ability}

In memory devices using different electrical and optical properties in the crystalline and amorphous states, a lower melting point is preferable, because the crystalline state can be converted to the amorphous state by laser beam or electrical pulse with relatively lower energy. Moreover, those solids forming a solid solution are preferable, because phase separation or segregation does not occur in the transition from amorphous to crystalline state. The phase separation or segregation in crystallization processes cause degradation of memory devices.

The values of the initial temperature, $T_{\mathrm{c}}$, and peak temperature, $T_{\mathrm{p}}$, of crystallization for different compositions of $\mathrm{Te}-\mathrm{Bi}-\mathrm{Se}$ system at various heating rates, $\beta$, are also obtained from the same thermograms. The kinetic resistance to crystallization is higher for larger differences between $T_{\mathrm{c}}$ and $T_{\mathrm{g}}$. The difference between $T_{\mathrm{c}}$ and $T_{\mathrm{g}}$, which is an indication of thermal stability of the glasses, decreases rapidly with the increase in $\mathrm{Bi}$ content for any given heating rate and the behaviour is similar at other heating rates.

For a memory/switching material, the thermal stability and ease of glass formation are of crucial importance. The values of $\left(T_{\mathrm{c}}-T_{\mathrm{g}}\right)$ are found to decrease with increase in $\mathrm{Bi}$ concentration. But these values are also found to increase with the increase in heating rates from $10 \mathrm{~K} / \mathrm{min}$ to $20 \mathrm{~K} / \mathrm{min}$. This indicates a decrease in thermal stability of glass (Mahadevan et al 1986) with an increase in bismuth concentration in the Te-Bi-Se glass system. The glass forming ability can be calculated using the following relations (Hurby 1972)

$$
K_{\mathrm{gl}}=\frac{T_{\mathrm{c}}-T_{\mathrm{g}}}{T_{\mathrm{m}}-T_{\mathrm{c}}} .
$$

The values of $K_{\mathrm{gl}}$ are found to decrease from 0.749 to 0.480 with an increase in $\mathrm{Bi}$ content from $28 \%$ to $38 \%$. Figure 5 shows the variation of $K_{\mathrm{gl}}$ with $\mathrm{Bi}$ content. It is

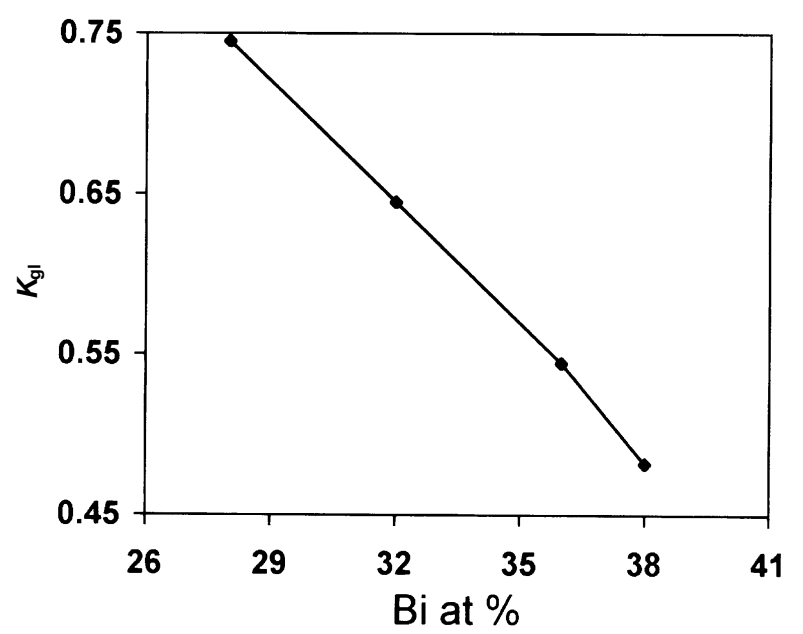

Figure 5. Variation of $K_{\mathrm{gl}}$ with Bi content.

noticed that the glasses with lower Bi content are easy to form compared to those with higher Bi content.

\section{Conclusions}

The glass transition temperature, $T_{\mathrm{g}}$, increases slightly with the variation of $\mathrm{Bi}$ content from $28 \%$ to $38 \%$. The value of peak of crystallization, $T_{\mathrm{p}}$, is found to decrease with the increase in $\mathrm{Bi}$ content. From the heating rate dependence of $T_{\mathrm{g}}$ and $T_{\mathrm{p}}$, the activation energy for the glass transition has been evaluated. The results are discussed on the basis of Kissinger's approach for non-isothermal crystallization. The values of activation energies for glass transition, $E_{\mathrm{t}}$, were found to decrease with increase in Bi content. The values of activation energies, using two different methods, are in good agreement with each other. So it can be concluded that any of these two methods can be used to calculate glass transition activation energy. Thermal stability of these glasses is also found in good command to form the glasses with ease. It is concluded that the glasses with lower $\mathrm{Bi}$ content are easy to form compared to those with higher $\mathrm{Bi}$ content.

\section{Acknowledgements}

The authors are highly indebted to University of Delhi, Delhi, for providing research facilities, MET trust members and Prof. R B Saxena, Director, MIT, Moradabad, for constant encouragement and kind guidance.

\section{References}

Afify N 1990 J. Non-Cryst. Solids 126130

Gosain D P, Shimizu T, Ohumura M, Suzuki M, Bando T and Okano S 1991 J. Mater. Sci. 263271

Hurby A 1972 Czech. J. Phys. B22 1187 
Ilyas M, Zulfequar M and Husain M 2000 Opt. Mater. 13397

Khan Z H, Zulfequar M and Husain M 1997 J. Mod. Opt. 4455

Kissinger H E 1956 J. Res. Nat. Bur. Stand. 57217

Lasocka M 1976 Mater. Sci. \& Engg. 23173

Mahadevan S, Giridhar A and Singh A K 1986 J. Non-Cryst. Solids $\mathbf{8 8} 11$

Onozuka A and Oda O 1988 J. Non-Cryst. Solids 103289

Rahman S, Ramana M V and Sivarama Sastry G 1991 J. Mater. Sci. Letts 10192
Saxena M, Rastogi N, Shukla S K and Bhatnagar P K 2001 Int. w. phy. semicond devices proc. (New Delhi: IWPSD) pp 1208 1211

Saxena M, Rastogi N, Agarwal A and Bhatnagar P K 2003 Indian J. Pure \& Appl. Phys. 41171

Tokunaga M, Nozawa Y, Iwasaki K, Tanigawa S and Harada $\mathrm{H}$ 1989 J. Magn. Magn. Mater. 8080

Vacher R, Delsanti M, Peous J, Cecchi L, Winter A and Zarzycki J 1974 J. Mater. Sci. 9829 